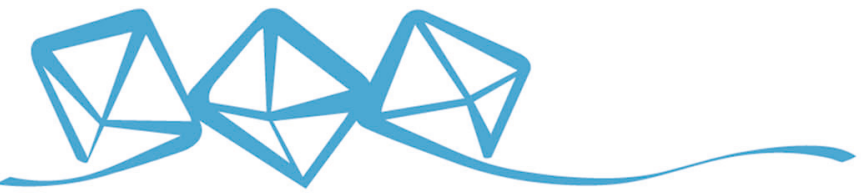 COMMUNICATIONS MATERIALS
}

ARTICLE

https://doi.org/10.1038/s43246-020-00084-0

OPEN

\section{Enhanced hole injection assisted by electric dipoles for efficient perovskite light-emitting diodes}

Xiangtian Xiao 1,2,3,5, Kai Wang (1) 1,2,5凶 , Taikang Ye ${ }^{1,2,5}$, Rui Cai ${ }^{1,2}$, Zhenwei Ren ${ }^{3}$, Dan Wu ${ }^{1,2,4}$, Xiangwei Qu ${ }^{1,2}$, Jiayun Sun ${ }^{1,2,3}$, Shihao Ding ${ }^{1,2}$, Xiao Wei Sun (1) ${ }^{1,2} \&$ Wallace C. H. Choy (i) ${ }^{3 凶}$

Enhanced hole injection is essential to achieve high performance in perovskite light-emitting diodes (LEDs). Here, a strategy is introduced to enhance hole injection by an electric dipole layer. Hopping theory demonstrates electric dipoles between hole injection layer and hole transport layer can enhance hole injection significantly. $\mathrm{MoO}_{3}$ is then chosen as the electric dipole layer between PEDOT:PSS (hole injection layer) and PVK (hole transport layer) to generate electric dipoles due to its deep conduction band level. Theoretical results demonstrate that strong electric fields are produced for efficient hole injection, and recombination rate is substantially increased. Capacitance-voltage analyses further prove efficient hole injection by introducing the electric dipole layer. Based on the proposed electric dipole layer structure, perovskite LEDs achieve a high current efficiency of $72.7 \mathrm{~cd} \mathrm{~A} \mathrm{~A}^{-1}$, indicating that electric dipole layers are a feasible approach to enhance perovskite LEDs performance.

\footnotetext{
${ }^{1}$ Shenzhen Key Laboratory for Advanced Quantum Dot Displays and Lighting, Department of Electrical and Electronic Engineering, Southern University of Science and Technology, Shenzhen 518055, China. ${ }^{2}$ Guangdong University Key Lab for Advanced Quantum Dot Displays and Lighting, Southern University of Science and Technology, Shenzhen 518055, China. ${ }^{3}$ Department of Electrical and Electronic Engineering, The University of Hong Kong, Hong Kong, China. ${ }^{4}$ Academy for Advanced Interdisciplinary Studies, Southern University of Science and Technology, Shenzhen 518055, China. ${ }^{5}$ These authors contributed equally: Xiangtian Xiao, Kai Wang, Taikang Ye. ${ }^{\circledR}$ email: wangk@sustc.edu.cn; chchoy@eee.hku.hk
} 
R ecently, metal halide perovskites have shown considerable potential for applications in optoelectronic devices, including solar cells, light-emitting diodes (LEDs), photodetectors, and lasers ${ }^{1-4}$. Especially, tremendous attentions have been paid in perovskite nanocrystals due to their excellent optical properties such as an easily tunable bandgap, high photoluminescence (PL) quantum yield (PLQY), and narrow-band emissions $^{5-9}$. These superior properties promote perovskite nanocrystals competitive candidates for LEDs. Green- and redNC PeLEDs have reached external quantum efficiency (EQE) exceeding $15 \%{ }^{2,10}$. Although EQE of thin film and quasi-twodimensional perovskite-based PeLEDs significantly increased to $>20 \%{ }^{11-13}$. Compared with the high PLQY of perovskite nanocrystals, there is still much room to improve their device performances.

To obtain high performance of PeLED, it is vital to balance the injection of holes and electrons. Otherwise, imbalanced charge injection will lead to the accumulation of the major carrier and the quenching of excitions, which will result in a poor efficiency, as well as the degradation of the functional layers ${ }^{14}$. To avoid mass carrier accumulation in devices, first, proper materials should be carefully selected with suitable energy level for the hole (or electron) injection layer. In general, the difference of energy level below $0.4 \mathrm{eV}$ can be considered as ohmic contact for the direct contact layers ${ }^{15}$, which will benefit for the charge transport. Second, the thickness of charge transport layers needs to be optimized to balance electron and hole transportation so that the charge can recombine effectively in the emission layer. The thickness of the transport layer can be optimized during the film fabrication process by controlling the parameters of spin-coating or evaporation speed and time, which are widely used to obtain the ideal film thickness in fabrication of the devices. Besides these methods, charge block layer has been also commonly adopted to reduce the major carrier transport rate to balance the charge injection. Zhang et al. ${ }^{14}$ inserted a thin polyvinyl pyrrolidone (PVP) buffer layer between the $\mathrm{ZnO}$ and perovskite layers. The PVP layer blocked excess electrons injection into perovsikte layer, balancing the injection of electrons and holes. Besides, Wang et al. ${ }^{16}$ introduced polyethylenimine ethoxylated between $\mathrm{ZnO}$ and perovskite layers, leading to a better charge balance and energy-level alignment. In addition, Dai et al. ${ }^{17}$ and Lin et al. ${ }^{11}$, respectively, introduced polymethyl methacrylate (PMMA) in quantum-dot-based LED and PeLED devices. The continuous PMMA film availability restrained the injection of excess electrons and optimized charge balance ${ }^{11,17}$.

However, these works mainly focus on blocking of electrons to realize the carrier balance. In a typical PeLED device structure indium tin oxide (ITO)/hole injection layer (HIL)/hole transport layer (HTL)/perovskite/electron transport layer (ETL)/LiF/Al, wherein poly(3,4-ethylenedioxythiophene):poly (styrenesulfonate) (PEDOT:PSS) is commonly used as HIL, Poly(9-vinylcarbazole)

a

Electric field caused by applied voltage

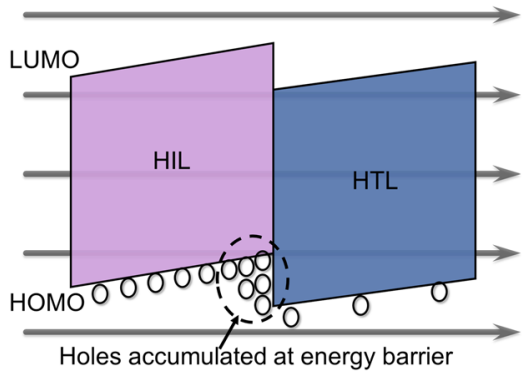

(PVK) is selected as HTL and 2,2',2'-(1,3,5-Benzinetriyl)-tris(1phenyl-1-H-benzimidazole) (TPBi) works as ETL. In this structure, the electron mobility of ETL is higher than the hole mobility of HTL. Therefore, electron injection rate is much faster than holes and cause imbalance charge injection ${ }^{14}$. Indeed, introducing an electron block layer at the interface of perovskite/ETL can retard the electron injection and improve the efficiency, while there is a large difference of highest occupied molecular orbital (HOMO) between PEDOT:PSS and PVK, which causes a nonnegligible hole injection barrier and results in a large energy loss and high turn-on voltage ${ }^{18-20}$. In fact, holes are minority carriers in the PeLED ${ }^{14}$; enhancing the hole injection rate will promote a balance of carrier injection, which will increase the quantity of recombined carriers for efficient electroluminescence (EL). Therefore, it is also essential to increase the hole injection rate for high PeLED performance. Several strategies have been implemented to enhance hole injection; for instance, inserting an interface layer between HTL and emission layer to adjust the HOMO level of HTL, and reduce hole injection barrier ${ }^{17,21}$. Besides, doping of HIL and HTL are also an effective method to increase their mobility so as to increase the hole injection rate re $^{22,23}$.

In this work, an efficient new strategy by introducing an electric dipole layer between HIL and HTL is proposed to enhance the hole injection. First, the field-dependent hole hopping theory has been introduced, which demonstrates that the electric dipoles between HIL and HTL can significantly enhance hole injection rate. Then, $\mathrm{MoO}_{3}$ is chosen as the interface layer between PEDOT:PSS and PVK to form electric dipoles for its deep conduction band level. Meanwhile, we theoretically analyze the distribution of electric fields, carrier density, and charge recombination rate in the device, which firmly demonstrates that the electric dipole layer of $\mathrm{MoO}_{3}$ can produce strong electric fields for enhancing the hole injection from PEDOT:PSS to PVK. Moreover, the low-frequency capacitance-voltage $(C-V)$ measurement and analysis further prove the more efficient hole injection by introducing the electric dipole layer of $\mathrm{MoO}_{3}$. With the advanced device structure of ITO/PEDOT:PSS/ $\mathrm{MoO}_{3} / \mathrm{PVK} /$ Perovskite/TPBi/LiF/Al, we have fabricated the PeLED with an improved EQE by $8.7 \%$ up to $16.8 \%$ and an improved current efficiency (CE) by $37.2 \mathrm{~cd} \mathrm{~A}^{-1}$ up to $72.7 \mathrm{~cd} \mathrm{~A}^{-1}$, indicating a feasible approach with the electric dipole layer of $\mathrm{MoO}_{3}$ to achieve a high PeLED performance.

\section{Results and discussion}

Electric dipole and field-dependent hole hopping theory. An electric dipole is a system consisting of a positive and a negative charge of equal magnitude, and separated by a small distance. To understand the effect of electric dipole on hole transport in PeLEDs, we study a typical hole transport model with the structure of HIL/HTL. Figure 1a shows a classicial HIL/HTL interface without an electric dipole layer. In this structure, the

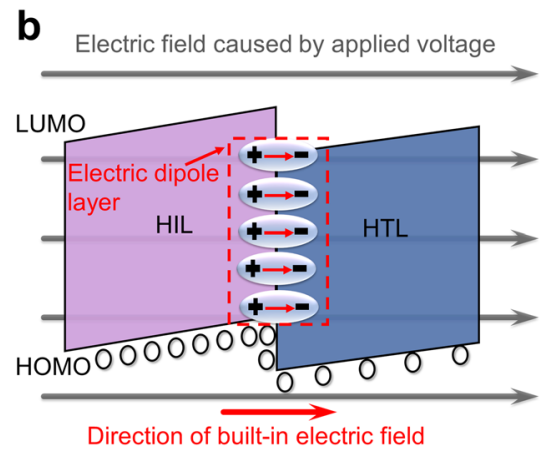

Fig. 1 Holes transporting under applied voltage. a Transporting by conventional HIL/HTL structure. b Transporting assisted by electric dipole layer. 
HOMO level of HTL is often deeper than that of HIL. Thererfore, there is an energy barrier between HIL and HTL. Under the applied forward voltage, holes are transported from HIL to HTL through their HOMO level. Due to the existence of energy barrier, part of the holes will be accumulated in this region. To improve the hole transportation, electric dipole layer is introduced. As is shown in the dotted box in Fig. 1b, an electric dipole layer, which consists of numerous electric dipoles, is applied on the interface between HIL and HTL. The dipole moment of these electric dipoles keeps the same direction, because in the presence of the electric field from HIL to HTL, a dipole tends to align itself parallel to the concerned field. The Coulomb force is existed between a positive and a negative charge in every electric dipole. According to the relation between Coulomb force $F$ and the electric field $E: E=F / q$ ( $q$ is the charge quantity), an built-in electric filed is formed in every electric dipole. Driven by the extra built-in electric field generated from the aligned electric dipoles, holes will be transported from HIL to HTL more efficiently.

In modeling, Miller-Abrahams theory is adopted to study the field-dependent hole hopping process, which is an effective method to describe the carriers hopping ${ }^{24,25}$. According to this theory, the parameter of the averaged hopping frequency $\omega\left(F_{\mathrm{e}}\right)$, which indicates the hole transporting rate from HIL to HTL, can be written as:

$$
\begin{gathered}
\omega\left(F_{\mathrm{e}}\right)=v_{0} \frac{\psi\left(F_{\mathrm{e}}\right)}{2 \gamma a} \exp \left[-\frac{H_{\mathrm{h}}^{\prime}}{k T}+\frac{1}{2}\left(\frac{\sigma}{k T}\right)-2 \gamma a\right], \\
\psi\left(F_{\mathrm{e}}\right)=\left[\frac{\exp \left(e F_{\mathrm{e}} a / k T\right)}{1-e F_{\mathrm{e}} / 2 \gamma k T}-1\right] \frac{k T}{e F_{\mathrm{e}} a},
\end{gathered}
$$

where $F_{\mathrm{e}}$ is the force caused by such a built-in electric field that shown in Fig. 1b, $\psi\left(F_{\mathrm{e}}\right)$ is the field-dependent factor, $a$ is the minimal hopping distance, $N$ is the concentration of hopping site, $v_{0}$ is the attempt-to-jump frequency, $H_{\mathrm{h}}^{\prime}$ is the energy barrier, $\sigma$ is the variance of the Gaussian energetic distribution of hopping states, $\gamma$ is the inverse localization radius of tunneling particles, $k$ is Boltzmann's constant, and $T$ is the temperature. For the fielddependent factor $\psi\left(F_{\mathrm{e}}\right)$, it will approach unity when electric field is low. When $F_{\mathrm{e}}>k T / e a$, the average hopping frequency becomes exponential. Therefore, the hole injection will be significantly enhanced due to high hopping frequency. As a result shown in Fig. 2b, compared with classical HIL/HTL structure, there are fewer holes accumulated at energy barrier, and more holes transported from HIL into HTL. Since this strong electric field can be formed by electric dipoles, how to build an electric dipole layer between HIL and HTL becomes a key issue.

With the understanding, $\mathrm{MoO}_{3}$ is proposed to realize such a suitable electric dipole layer. To understand the mechanism of the formed electric dipole layer, a typical structure of PeLED is

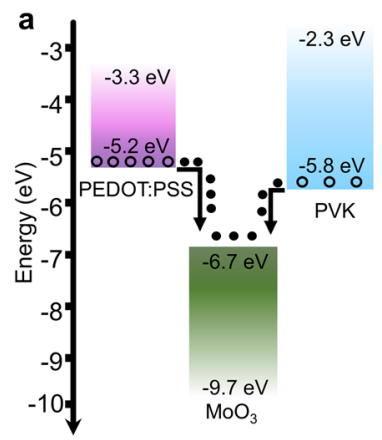

b

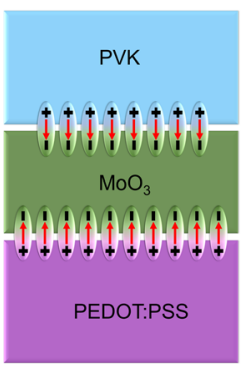

Fig. 2 Electric dipoles generated by carriers transfer. a Schematic of carriers transfer in PEDOT:PSS/MoO$/ \mathrm{PVK}$ structure. b Electric dipoles generated at PEDOT:PSS/ $\mathrm{MoO}_{3}$ and $\mathrm{MoO}_{3} / \mathrm{PVK}$ interface layer. considered here: ITO/ PEDOT:PSS/ $\mathrm{MoO}_{3} / \mathrm{PVK} /$ Perovskite/TPBi/ $\mathrm{LiF} / \mathrm{Al}$. As the key component, the electric dipole is realized by an extremely thin $\mathrm{MoO}_{3}$ layer between PEDOT:PSS and PVK. Figure 2 describes the schematic of carriers transfer of devices with the $\mathrm{MoO}_{3}$ interlayer. Figure 2a shows the alignments of the HOMO of PEDOT:PSS and PVK, and the conduction band minimum (CBM) of $\mathrm{MoO}_{3}$. Without any bias, there are numerous electron-hole pairs in the HOMO of PEDOT:PSS and PVK. Due to the extremely deep CBM of $\mathrm{MoO}_{3}(-6.7 \mathrm{eV})$, some electrons in those electron-hole pairs will transfer to $\mathrm{MoO}_{3}$ and be trapped in such a "well" caused by $\mathrm{MoO}_{3}$. This transfer is spontaneous without any external voltage bias. It will lead to two results, which are shown in Fig. 2b: (1) every transported electron in $\mathrm{MoO}_{3}$ has a corresponding hole in PEDOT:PSS or PVK, and electric dipoles are thus formed at the interfaces due to the electron-hole pairs, forming two groups of electric dipoles with opposite built-in electric fields. Furthermore, the difference between HOMO of PEDOT:PSS and CBM of $\mathrm{MoO}_{3}(1.5 \mathrm{eV})$ is larger than that between PVK and $\mathrm{MoO}_{3}(0.9 \mathrm{eV})$. Therefore, there are more electrons transferred from PEDOT:PSS to $\mathrm{MoO}_{3}$ and the number of electric dipoles at PEDOT:PSS/ $/ \mathrm{MoO}_{3}$ interface is more than that at $\mathrm{PVK} / \mathrm{MoO}_{3}$ interface. Meanwhile, there are more holes in $p$-type PEDOT:PSS than those in PVK (nearly neutral $)^{26,27}$. These reasons lead to a stronger electrical field at PEDOT:PSS $/ \mathrm{MoO}_{3}$ interface than that at $\mathrm{PVK} / \mathrm{MoO}_{3}$ interface, facilitating the injection of holes from PEDOT:PSS to PVK. (2) Owing to the transfer of electrons, the hole concentration is increased both in PEDOT:PSS and PVK. As the current density of holes inside the HTL is defined as a product of the spatial density of holes $\rho(x)$, electric field strength $F$, and hole mobility $\mu$ :

$$
J_{\mathrm{h}}=\mu \rho(x) F(x),
$$

Therefore, higher hole concentrations in PEDOT:PSS and PVK will obviously increase the hole current, which means the hole injection can be enhanced, and better hole-electron balance can be realized in PeLED. In addition, the VBM of $\mathrm{MoO}_{3}$ is extraordinary deep $(-9.7 \mathrm{eV})$. Due to the modification of $\mathrm{MoO}_{3}$, the work function of PEDOT:PSS will be increased to realize a better energy-level alignment with $\mathrm{PVK}^{12}$.

Simulation. To further support above theory and discussion, theorical analyses of charge density, electric field and recombination rate of a $\mathrm{MoO}_{3}$-based PeLED were conducted through simulation and calculations. Here, the well-established physical drift-diffusion model was used, which is consisted of the continuity equation for electrons and holes coupled with the Poisson equation. Furthermore, field-dependent Miller-Abrahams theory was also considered to research hole hopping between PEDOT: PSS, $\mathrm{MoO}_{3}$, and PVK interfaces. Formula 1, 2, and related parameters were implemented in the simulation.

Figure 3a shows the device structure of PeLED for simulation analysis, which consists of ITO $(100 \mathrm{~nm})$, PEDOT:PSS $(20 \mathrm{~nm})$, $\mathrm{MoO}_{3}(1.5 \mathrm{~nm})$, PVK $(40 \mathrm{~nm}), \mathrm{FAPb}_{0.7} \mathrm{Sn}_{0.3} \mathrm{Br}_{3}$ perovskite $(20$ $\mathrm{nm})$, TPBi $(45 \mathrm{~nm})$, and LiF $(1 \mathrm{~nm}) / \mathrm{Al}(100 \mathrm{~nm})$. Here, PEDOT: PSS is HIL, PVK is HTL, perovskite is the active layer, and TPBi is ETL. The alignment of energy levels of the different components in the device is shown in Fig. 3b. Figure 3c shows the distribution of charge density in PEDOT:PSS, $\mathrm{MoO}_{3}$, and PVK. Due to carriers transfer as mentioned above, some electrons in PEDOT:PSS and PVK move into $\mathrm{MoO}_{3}$, increasing the hole densitys both in PEDOT:PSS and PVK around $\mathrm{MoO}_{3}$ interfaces. Moreover, the PEDOT:PSS has a weak p-type doping; as a result, the density of holes in PEDOT:PSS is higher than that in PVK. In Fig. 3d, two electric fields, which are in opposite directions, were formed at the PEDOT:PSS/ $\mathrm{MoO}_{3}$ interface, as well as the 

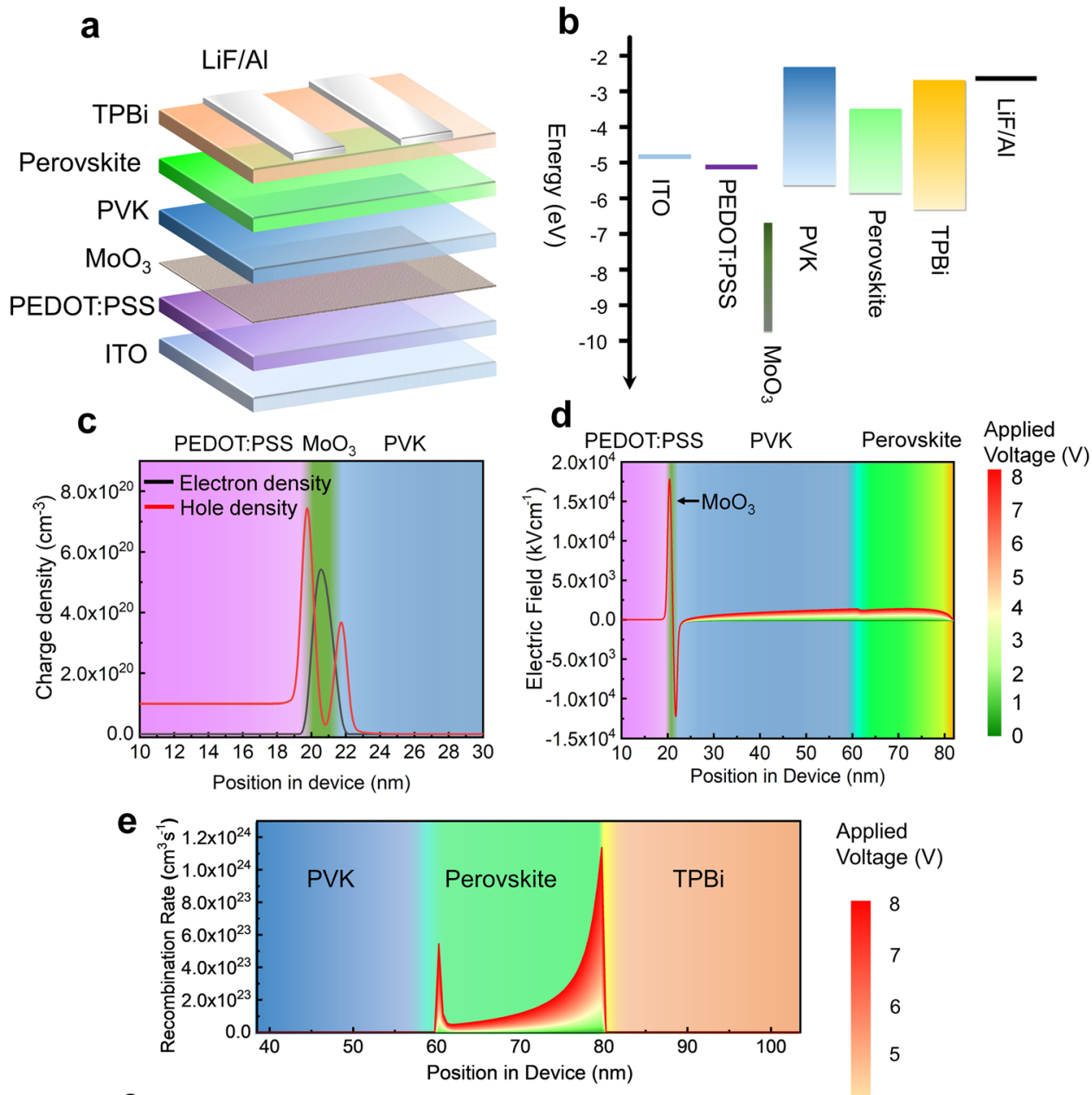

Applied

Voltage (V)
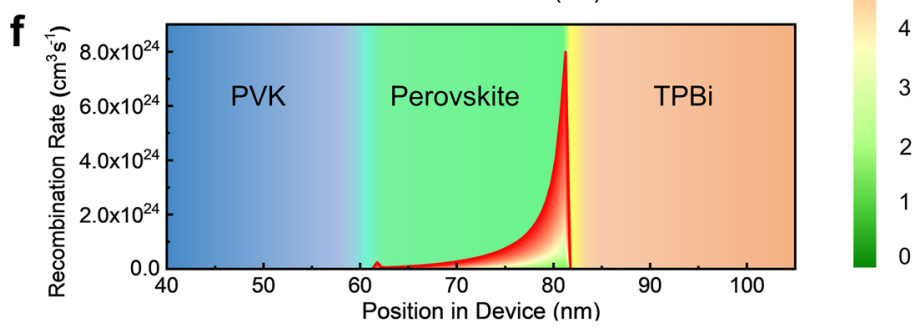

Fig. 3 Simulation results of PeLEDs. a Device structure of PeLED for simulation. $\mathbf{b}$ Energy-level alignment of the proposed structure. c Distribution of charge density in the device. $\mathbf{d}$ Distribution of electric field in the device. e Distribution of recombination rate in the device without $\mathrm{MoO}_{3}$. $\mathbf{f}$ Distribution of recombination rate in the device with $\mathrm{MoO}_{3}$.

$\mathrm{MoO}_{3} / \mathrm{PVK}$ interface with different applied voltages from $0 \mathrm{~V}$ to $8 \mathrm{~V}$. Electric fields distribution with specific applied voltage 0,2 , 4 , and $6 \mathrm{~V}$ are shown in Supplementary Fig. 1. Take the direction of hole transportation as the forward direction. As the intensity of positive electric field is stronger than that of negative electric field, the positive electric field between PEDOT:PSS and $\mathrm{MoO}_{3}$ leads to a higher field-dependent factor. According to Eq. (1), it obviously increases averaged hopping frequency $\omega\left(F_{\mathrm{e}}\right)$ for holes hopping from HIL to HTL. Meanwhile, as the direction of the electric field between $\mathrm{MoO}_{3}$ and PVK is negative, it presents a resistance for holes hopping to PVK. According to Fig. 3d, the magnitude of positive electric field is higher than that of negative electric field by $5000 \mathrm{kV} \mathrm{cm}^{-2}$. Therefore, positive electric field is dominant on hole hopping process. In addition, the negative electric field presents a resistance for excess electrons that moving from perovskite layer to anode. Hence, this negative electric field would play a role, such as an electron blocking layer, and significantly block the excess electrons. Overall, by generating electric dipoles at the interfaces, hole hopping from PEDOT:PSS to PVK is enhanced and excess electrons are more effectively blocked, realizing a better balance of carrier injection. In addition, the better charge balance can be further confirmed by the distribution of charge density in perovskite layer as shown and discussed in Supplementary Fig. 2.

Figure $3 \mathrm{e}$ shows the distribution of the recombination rate in the device without $\mathrm{MoO}_{3}$ interface layer. There are two recombination peaks: (i) the weak one, with the peak rate of $5.4 \times 10^{23} \mathrm{~cm}^{3} \mathrm{~s}^{-1}$, is located near the perovskite/PVK interface and (ii) the strong one, with the peak rate of $1.1 \times 10^{24} \mathrm{~cm}^{3} \mathrm{~s}^{-1}$, is located near the perovskite/TPBi interface. This distribution of recombination peaks is mainly decided by the mobilities of HTL and ETL, and the hole injection barrier between HIL and HTL. According to Supplementary Table 2, under the operation voltage of PeLEDs, mobility of PVK $\left(10^{-6} \mathrm{~cm}^{2} \mathrm{~V}^{-1} \mathrm{~s}^{-1}\right)$ is lower than that of TPBi $\left(10^{-4} \mathrm{~cm}^{2} \mathrm{~V}^{-1} \mathrm{~s}^{-1}\right)$, as well as considering the large hole injection barrier between HIL and HTL, hole injection is 
obviously weaker than that of electrons (Supplementary Fig. 11). Mobility of PVK $\left(10^{-6} \mathrm{~cm}^{2} \mathrm{~V}^{-1} \mathrm{~s}^{-1}\right)$ is lower than that of TPBi $\left(10^{-4} \mathrm{~cm}^{2} \mathrm{~V}^{-1} \mathrm{~s}^{-1}\right)$, as well as considering the large hole injection barrier between HIL and HTL, hole injection is obviously weaker than that of electrons. Therefore, some excess electrons will go across perovskite layer and recombined with holes at PVK/ perovskite interface. As a result, there is still a recombination peak at this interface in Fig. 3e. Figure $3 \mathrm{f}$ shows the distribution of recombination rate with $\mathrm{MoO}_{3}$ interface layer. Compared with the result in Fig. 3e, the recombination rate near the perovskite/ TPBi interface is significantly increased to $8.0 \times 10^{24} \mathrm{~cm}^{3} \mathrm{~s}^{-1}$ (more than sevenfold increase).

Meanwhile, the weak recombination peak, which is near the perovskite/PVK interface, is obviously restrained (only $2.4 \times$ $10^{23} \mathrm{~cm}^{3} \mathrm{~s}^{-1}$ ). These optimizations of recombination rate attribute to the introduction of $\mathrm{MoO}_{3}$ interface layer, which generates electric dipoles at the interfaces of HIL/HTL, facilitating holes hopping from HIL to HTL and at the same time enhancing the hole concentrations both at HIL and HTL. Therefore, holes transport into perovskite layer is increased, while electrons transport into perovskite layer remains unchanged. Since the hole is the minority carrier in PeLED, the increase of hole injection helps realize a better balance of carrier injection. Compared with the PeLED without $\mathrm{MoO}_{3}$ interface layer, in the improved PeLED, more holes will have recombinations with more electrons near the perovskite/TPBi interface. Meanwhile, as more electrons are consumed near the perovskite/TPBi interface, fewer electrons diffuse to the perovskite/PVK interface, restraining recombination at this interface.

PeLED with $\mathrm{MoO}_{3}$ interface layer. The PeLEDs were fabricated and measured to demonstrate the enhancement of hole injection. Typical device structure was kept the same with that in the simulation section. As is shown in the schematic diagram in Fig. 4a, the proposed PeLED device consisted of spin-coated layer including PEDOT:PSS, PVK, $\mathrm{FAPb}_{0.7} \mathrm{Sn}_{0.3} \mathrm{Br}_{3}$ perovskite nanocrystals, and the evaporated layers including $\mathrm{MoO}_{3}, \mathrm{TPBi}$, and $\mathrm{LiF} / \mathrm{Al}$. The $\mathrm{MoO}_{3}$ layer was inserted between the PEDOT: PSS and PVK layer. Though this $\mathrm{MoO}_{3}$ layer was very thin, it was still intact after deposition of successive PVK layer (Supplementary Fig. 9). Compact thin films with few pinholes are formed when $\mathrm{MoO}_{3}$ layer is evaporated on PEDOT:PSS film or not (Supplementary Fig. 3). Here, the optimized thickness of $\mathrm{MoO}_{3}$ was determined as $1.5 \mathrm{~nm}$ (Supplementary Fig. 4). When the thickness of $\mathrm{MoO}_{3}$ layer was $<1.5 \mathrm{~nm}, \mathrm{MoO}_{3}$ was too thin to realize the best performance on electron collection, then electric dipoles could not form a strong enough electric field to enhance hole hopping. In addition, it could not help reducing the energy barrier between PEDOT:PSS and PVK sufficiently. When the thickness of $\mathrm{MoO}_{3}$ layer was too large, it could form a compact film on PEDOT:PSS. In this case, hole hopping from PEDOT:PSS to PVK could hence be weak due to the long minimal hopping distance.

The high-binding energy of secondary-electron cutoff ( $\left.E_{\text {cutoff }}\right)$ and the onset energy in VB regions $\left(E_{\text {onset }}\right)$ are extracted from the ultraviolet photoelectron spectroscopy spectra as shown in Fig. $4 \mathrm{~b}$. By using the equation of $\mathrm{VB}=21.2 \mathrm{eV}-\left(E_{\text {cutoff }}-E_{\text {onset }}\right)$, the HOMO levels of PEDOT:PSS with and without $\mathrm{MoO}_{3}$ are estimated to be -5.5 and $-5.2 \mathrm{eV}$, respectively. As the HOMO level of PVK is $-5.8 \mathrm{eV}$, hole injection barrier between the PEDOT:PSS (HIL) and PVK (HTL) is hence decreased from 0.6 to $0.3 \mathrm{eV}$ by introducing $\mathrm{MoO}_{3}$ layer. Therefore, the $\mathrm{MoO}_{3}$ interface layer could not only form electric dipole layer but also realized better energy-level alignment to help hole transporting. Meanwhile, the change of binding energy also indicated the shift of vaccum level (Supplementary Fig. 15), which further proved that the interfacial electric dipole was formed. The performances of the PeLED based on $\mathrm{FAPb}_{0.7} \mathrm{Sn}_{0.3} \mathrm{Br}_{3}$ perovskite nanocrystals with and without $\mathrm{MoO}_{3}$ were displayed in Fig. 4c-f. Figure 4c shows the EL spectra of the proposed PeLED. The insert graph shows the pure and bright-light emission of PeLED devices at $6 \mathrm{~V}$ bias. Compared with the PL spectra of $\mathrm{FAPb}_{07} \mathrm{Sn}_{03} \mathrm{Br}_{3}$ perovskite solution, there is a red shift for EL from 527 to $531 \mathrm{~nm}$ due to the quantum-confined Stark effect ${ }^{28}$. The full width at half maximum remains almost unchanged at $22.5 \mathrm{~nm}$. Figure $4 \mathrm{~d}$ shows the current density-voltage $(J-V)$ and luminance-voltage $(L-V)$ characteristics. The $\mathrm{MoO}_{3}$-based PeLED shows much higher current density (more than double increase from 5 to $8.5 \mathrm{~V}$ ) and the maximum luminance is enhanced from 4000 to $14,700 \mathrm{~cd} \mathrm{~m}^{-2}$, which is a 3.7 times improvement. In addition, the turn-on voltage $\left(V_{\text {on }}\right)$ decreased from 3.6 to $2.8 \mathrm{~V}$ (Supplementary Fig. 8a) due to the reduced hole injection barrier and improved hole injection. As demonstrated in Fig. 4e, f, with the implementation of $\mathrm{MoO}_{3}$ interface layer, the EQE increases from $8.7 \%$ to $16.8 \%$. Meanwhile, the CE increases from 37.2 to $72.7 \mathrm{~cd}$ $\mathrm{A}^{-1}$. These improvements are mainly attributed to the enhanced hole injection, contributed from the effective hole hopping, and increased hole concentrations of HTL. Meanwhile, as shown in Supplementary Fig. 5, although $\mathrm{MoO}_{3}$ can also reduce hole injection barrier and slightly increase the luminance, it only makes a few contributions to the device performance. Therefore, electric dipole layer is the primary cause that leads to the significantly increasing of device performance. In addition, devices still demonstrated efficiency roll-off particularly at high voltage. The synchronous EL-PL measurement showed that PLQY decrease as well as the damage of transport layers at high voltage may lead to this result (Supplementary Figs. 13 and 14). As referenced by Supplementary Table 1, the CE in this work is one of the highest values in green PeLED that based on perovskite nanocrystals or quantum dots. Therefore, the enhancement of hole injection in PeLED by introducing electric dipoles through $\mathrm{MoO}_{3}$ has been theoretically analyzed, simulate calculated, and finally experimentally realized. Futhermore, due to the better charge balance, the PeLED with $\mathrm{MoO}_{3}$ also shows the longer operation time (Supplementary Fig. 6). Compared with the PeLED without $\mathrm{MoO}_{3}$, the device with $\mathrm{MoO}_{3}$ interface layer shows a nearly double increased the $\mathrm{T}_{50}$ lifetime, increased from 2.1 to 4 mins. There are three main reasons for the improvement of device stability as follows: (1) the turn-on voltage of the device is reduced from 3.6 to $2.8 \mathrm{~V}$. Therefore, the device can realize a high luminance under a relatively lower voltage. This lower voltage reduces the magnitude of electric field in the device and mitigates the ion migration. (2) Due to the existence of the $\mathrm{MoO}_{3}$ interface layer, the hole injection is enhanced, and the carrier balance becomes better, which help reduce the damage of excess carriers in the device. (3) This $\mathrm{MoO}_{3}$ interface layer can mitigate the concentration of electric dipoles for the HTL. Better reproducibility can be also realized with $\mathrm{MoO}_{3}$ interface layer (Supplementary Fig. 7). In addition, compared with some other HTL materials with high mobility (e.g., poly[(9,9-dioctylfluorenyl-2,7-diyl)-alt(4,4'-(N-(4-butylphenyl)))]), PEDOT:PSS/ $\mathrm{MoO}_{3} /$ PVK structure shows better energy alignment and lower hole injection barrier (Supplementary Fig. 12). Therefore, choosing an ultra-thin $\mathrm{MoO}_{3}$ interface layer between HIL and HTL can better help improve the hole injection, and realize carrier balance of PeLEDs.

Low-frequency $C-V$ measurement. To further analyze the enhanced hole injection in the proposed PeLED, low-frequency $C-V$ measurement was developed. By measuring the differential 
a

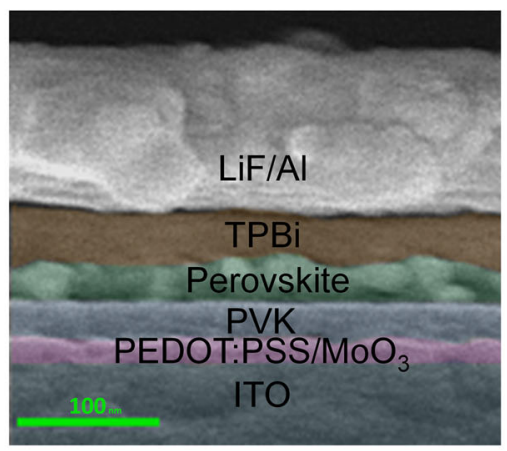

C
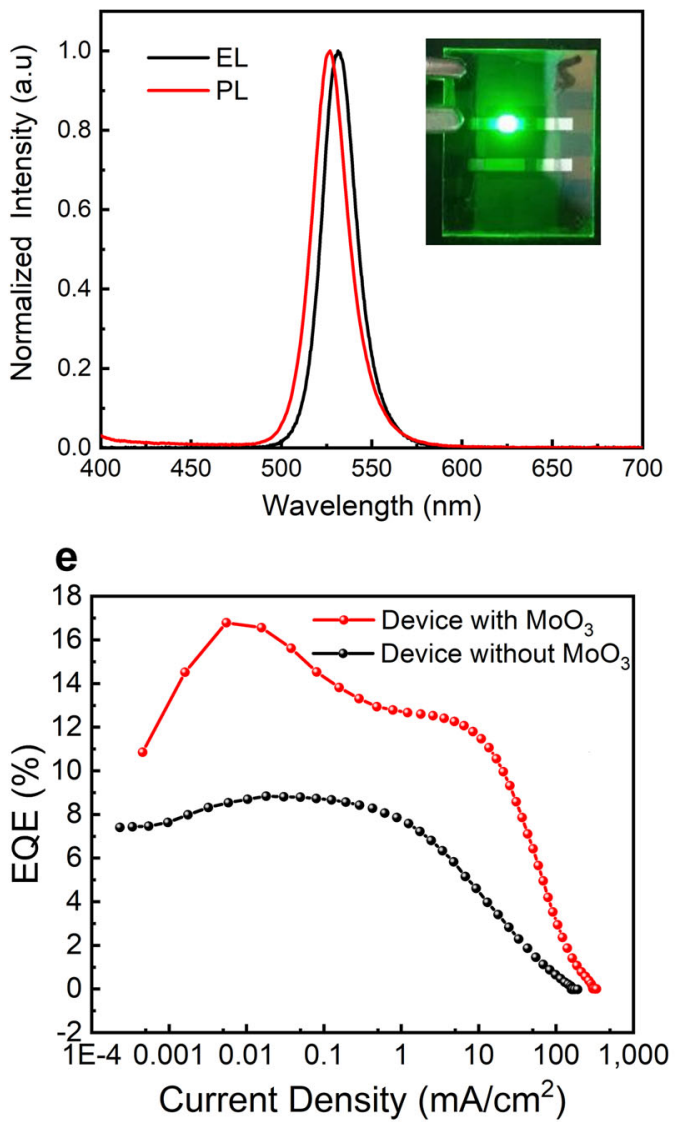

b
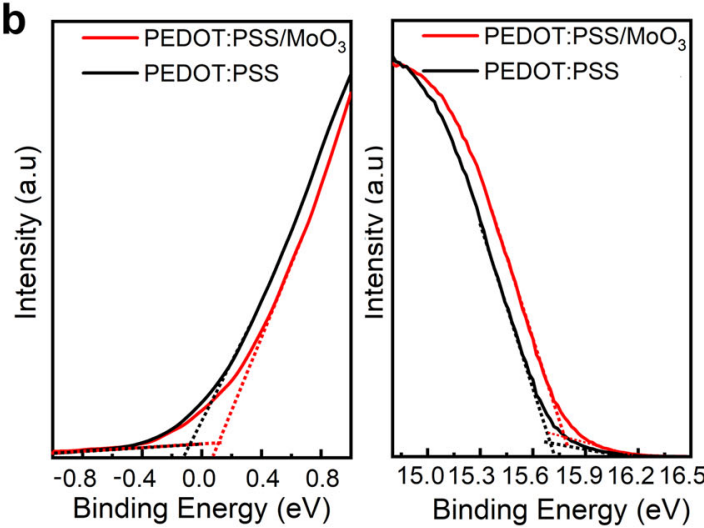

d

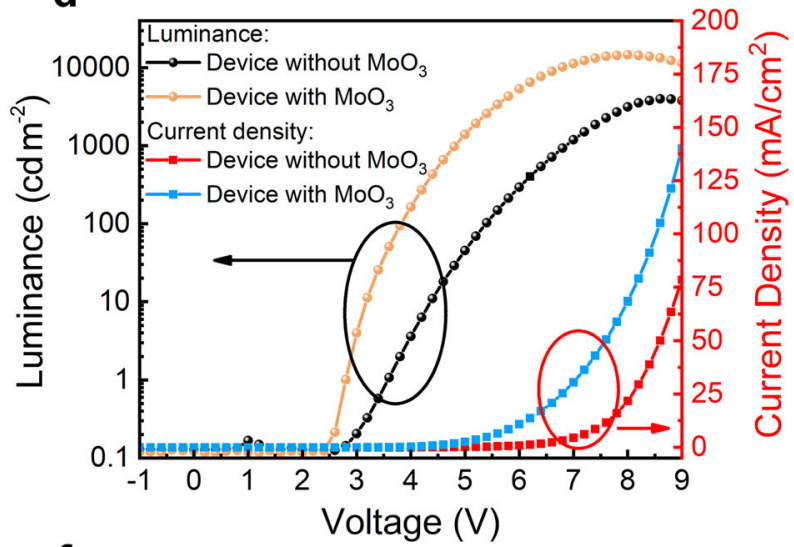

f

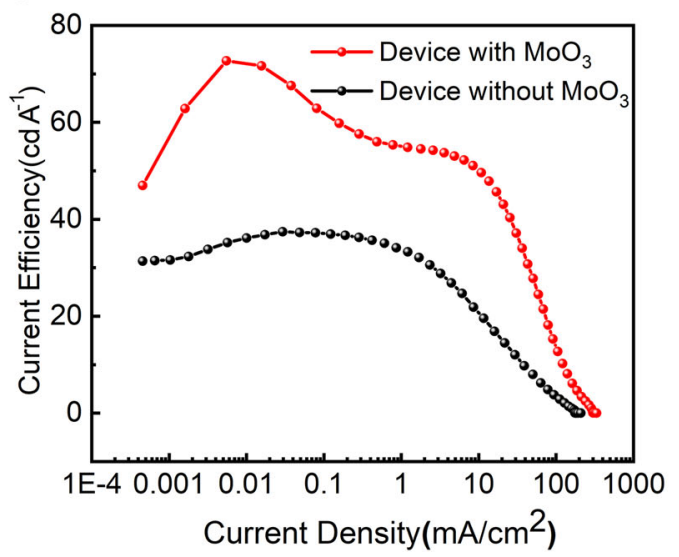

Fig. 4 Structure and EL performances of PeLEDs. a Cross-section SEM of the PeLED device. $\mathbf{b}$ The VB-edge region (left) and secondary-electron cutoff region (right) of PEDOT:PSS and PEDOT:PSS/ $\mathrm{MoO}_{3}$ structure. $\mathbf{c}$ The photoluminescence and electroluminescence (at $6 \mathrm{~V}$ bias) spectra. $\mathbf{d}$ The luminance and current density vs. the applied voltage $(L-V, J-V$ curve). e The external quantum efficiency vs current density (EQE curve). $\mathbf{f}$ The current efficiency vs the current density (CE curve).

capacitance, it can calculate out the change of charge quantity in PeLED devices by $d Q=C d V$. Therefore, the $C-V$ analysis is an effective way to evaluate the carrier injection, transporting, recombination and charge balance in PeLED devices. Here, relatively low modulation frequency of $1 \mathrm{KHz}$ was used to take many factors into consideration, including traps, transit time, and recombination rate. Therefore, details of carrier injection, transporting, and recombination could hence be better revealed ${ }^{29,30}$.

Interestingly, as shown in Fig. 5, $C-V$ results of PeLEDs with $\mathrm{MoO}_{3}$ interface layer (red curve) present a very different tendency with that of PeLEDs without $\mathrm{MoO}_{3}$ interface layer (black curve) at high bias voltage. When the voltage is low $(<2 \mathrm{~V})$, injected carriers in PeLEDs are few, so $C-V$ curves reflect the geometric capacitance of devices. In this case, the capacitance value is depended on materials themselves, so these two curves keep almost the same and unchanged value. When the voltage is increased from $2 \mathrm{~V}$, the capacitance increases significantly. Especially, red curve rises faster than the black curve. In this process, more and more carriers are injected in PeLEDs with the increase of applied voltage, and larger number of injected carriers lead to the faster rise rate. As there are the same device structure $\mathrm{TPBi} / \mathrm{LiF} / \mathrm{Al}$ for electron injection in PeLEDs, the electron injection with and without $\mathrm{MoO}_{3}$ keep unchanged. Therefore, faster rise of red curve can only be explained by the enhanced hole injection. When the applied voltage is more than $4 \mathrm{~V}$, there is a capacitance peak for these two curves. After this peak, red curve 


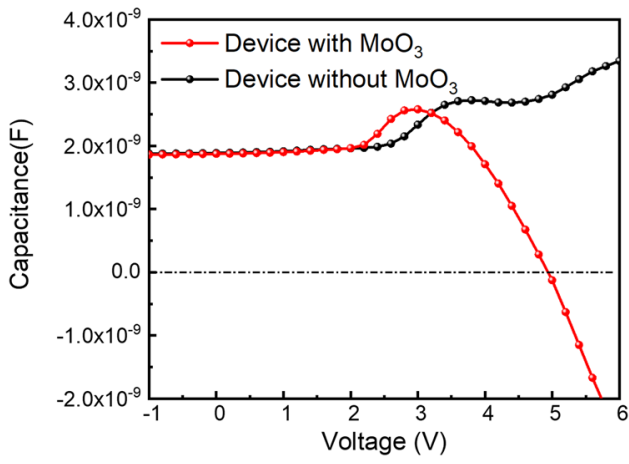

Fig. $5 \mathbf{C}-\boldsymbol{V}$ results of PeLEDs. Red and balck lines are devices with and without $\mathrm{MoO}_{3}$.

keeps declining, but black curve drops then rise again. To understand these tendencies, a basic formula to describe the capacitance is introduced ${ }^{30}$ :

$$
C=\frac{d Q}{d U}=\frac{d Q_{\text {in }}+d Q_{\text {trap }}-d Q_{\mathrm{r}}}{d V}
$$

where $Q_{\text {in }}, Q_{\text {trap }}$, and $Q_{r}$ is the total charge quantity of injected carriers, trapped carriers, and recombined carriers, respectively. As the only difference between PeLEDs in our experiment is the insertion of $1.5 \mathrm{~nm} \mathrm{MoO}_{3}, Q_{\text {trap }}$ would only make a small contribution, whereas $Q_{\text {in }}$ and $Q_{\mathrm{r}}$ are dominant in $C-V$ curves. Therefore, the capacitance peak is formed by the recombination of carriers. Furthermore, the tendency of curves after capacitance peak is directly depended on the recombination rate. If the recombination is strong enough, the $C-V$ curve would decline. Once there is an extremely strong recombination, which leads to $d Q_{\mathrm{r}}>d Q_{\text {in }}+d Q_{\text {trap }}$, the capacitance will become negative ${ }^{30}$. Negative capacitance of red curve indicates the high recombination efficiency of the PeLED with $\mathrm{MoO}_{3}$ interface layer. Meanwhile, the rising tendency of the black curve shows a poor recombination efficiency of the PeLED without $\mathrm{MoO}_{3}$ interface layer. These results coincides well with the $\mathrm{EQE}$ and luminance performance of PeLEDs. From our best knowledge, this is the first time of demonstrating the negative capacitance PeLED, indicating that strong recombination, which has already been obtained in quantum dot LED and organic LED demonstrating as negative capacitance, also can be achieved in PeLED by introducing an electric dipole layer between HIL and HTL.

\section{Conclusion}

In summary, we report a unique strategy by introducing a $\mathrm{MoO}_{3}$ electric dipole layer to enhance the hole injection for efficient PeLED. Theoretically, we demonstrate that the $\mathrm{MoO}_{3}$ interface layer can form positive electric fields across the interface of PEDOT:PSS $/ \mathrm{MoO}_{3} / \mathrm{PVK}$, which promotes the hole transport and eventually, notably improving the radiative recombination intensity of PeLED. With the electric dipole layer-based device structure of ITO/PEDOT:PSS/MoO $/ \mathrm{PVK} /$ Perovskite/TPBi/LiF/ $\mathrm{Al}$, we have fabricated the PeLED with an improved CE of $72.7 \mathrm{~cd} \mathrm{~A}^{-1}$, indicating a feasible approach with the electric dipole layer of $\mathrm{MoO}_{3}$ to achieve a high PeLED performance.

\section{Methods}

Material. We prepared the perovskite nanocrystals $\left(\mathrm{FAPb}_{1-x} \mathrm{Sn}_{x} \mathrm{Br}_{3}\right)$ according to ref. ${ }^{31}$. The host is popular $\mathrm{FAPbBr}_{3}$ nanocrystal, $\mathrm{FA}^{+}$has a larger ion radius than $\mathrm{CS}^{+}$and $\mathrm{MA}^{+}$ions, results a larger tolerant factor. Thus, there were a lot of cation mixed perovskite/nanocrystals based on $\mathrm{FAPbBr}_{3}$ and the device performance can be improved by cation mixed. In our experiment, we used Sn doping to adjust the optoelectronic properties of $\mathrm{FAPbBr}_{3}$ nanocrystals. In the mix-cation perovskite, the Sn replace the position of $\mathrm{Pb}$. By increasing the value of $x$ from 0 to 0.5 , the bandgap of perovskite nanocrystal increased from 2.30 to $2.38 \mathrm{eV}$. There was an enhancement of PL intensity with $x$ from 0 to 0.3 and the highest PLQYs was $97.5 \%$ at $x=0.2$. The improvement of PL is because of the electron state modulation by Sn doping. When $x=0.3$, the devices have the best performance, so we fabricated the PeLED devices based on $\mathrm{FAPb}_{0.7} \mathrm{Sn}_{0.3} \mathrm{Br}_{3}$ perovskite nanocrystals to do further optimization and analysis.

Device fabrication. The PeLED was fabricated on ITO-patterned glass substrates with a sheet resistance of $20 \Omega \mathrm{sq}^{-1}$. The cleaning ITO substrate was cleaned by sonication in soaps-water, deionized water, acetone and methanol for $20 \mathrm{~min}$ each, and then treated with ultraviolet ozone for $20 \mathrm{~min}$ before film deposition. PEDOT: PSS was spin-coated at 4000 r.p.m. (1500 r.p.m. $\mathrm{min}^{-1}$ accelerated speed) for $45 \mathrm{~s}$ then annealing at $130^{\circ} \mathrm{C}$ for $15 \mathrm{~min} . \mathrm{MoO}_{3}$ was deposited using thermal evaporation at a rate of $0.002 \mathrm{~nm} \mathrm{~s}^{-1}$ under high vacuum $\left(<5 \times 10^{-4} \mathrm{~Pa}\right)$. PVK $\left(10 \mathrm{mg} \mathrm{ml}^{-1}\right.$, in chlorobenzene) was spin-coated on top of $\mathrm{MoO}_{3}$ layer at 3000 r.p.m. (1500 r.p.m. $\min ^{-2}$ accelerated speed) for $45 \mathrm{~s}$, baked at $120^{\circ} \mathrm{C}$ for $10 \mathrm{~min}$. Perovskite nanocrystal was deposited on PVK layer at 1500 r.p.m. (1500 r.p.m. $\mathrm{min}^{-2}$ accelerated speed) for $45 \mathrm{~s}$ without annealing. Finally, TPBi $(45 \mathrm{~nm}), \operatorname{LiF}(1 \mathrm{~nm}), \mathrm{Al}(100 \mathrm{~nm})$ were deposited by thermal evaporation at rate of $0.05,0.001$, and $0.2 \mathrm{~nm} \mathrm{~s}^{-1}$, repectively, under high vacuum $\left(<5 \times 10^{-4} \mathrm{~Pa}\right)$.

Simulations. The electrical simulations were performed with Setfos 4.6 software, which solves the drift-diffusion equations for electron and holes, and considers exciton formation, mdiffusion, and decay in the framework of radiating dipoles inside amcavity including the Purcell effect. Either constant or field-dependent electron and hole mobilities according to the Poole-Frenkel model were used. The chosen HOMO/lowest unoccupied molecular orbital values are displayed in Fig. 3b and the boundary conditions for the charge carrier densities at the electrodes were set to satisfy the Fermi-level alignment at thermal equilibrium. Exciton generation is permitted only in the emission layer and follows the standard Langevin recombination

Measurement. The luminance area of our devices is $2 \times 2 \mathrm{~mm}^{2}$. The PLQY and PL spectra were measured by absolute PLQY spectrometer (Hamamatsu quantaurusQY C11347-12). The EL spectrum was measured by fiber optic spectrometer (Ocean optics USB 2000) in the forward of luminance area. We assume the device is lambert illuminant, and the current density-voltage-luminance curve was measured by a Keithley 2614B source and a PIN-25D silicon photodiode. All the tests were done in the air at room temperature.

\section{Data availability}

The data that support the finding of this study are available from the corresponding authors upon reasonable request.

Received: 7 May 2020; Accepted: 14 October 2020; Published online: 06 November 2020

\section{References}

1. Stoumpos, C. C. et al. Halide perovskites: poor man's high-performance semiconductors. Adv. Mater. 28, 5778-5793 (2016).

2. Adinolfi, V. et al. The electrical and optical properties of organometal halide perovskites relevant to optoelectronic performance. Adv. Mater. 30, 1700764 (2018).

3. Veldhuis, S. A. et al. Perovskite materials for light-emitting diodes and lasers. Adv. Mater. 28, 6804-6834 (2016)

4. Avigad, E. et al. Studying the effect of $\mathrm{MoO}_{3}$ in hole-conductor-free perovskite solar cells. ACS Energy Lett. 3, 2240-2245 (2018).

5. Han, D. et al. Efficient light-emitting diodes based on in situ fabricated $\mathrm{FaPbBr}_{3}$ nanocrystals: the enhancing role of the ligand-assisted reprecipitation process. ACS Nano 12, 8808 (2018).

6. Song, J. et al. Organic-inorganic hybrid passivation enables perovskite QLEDs with an EQE of 16.48\%. Adv. Mater. 30, 1805409 (2018).

7. Zhang, X. et al. Hybrid perovskite light-emitting diodes based on perovskite nanocrystals with organic-inorganic mixed cations. Adv. Mater. 29, 1606405 (2017)

8. Deng, W. et al. Organometal halide perovskite quantum dot light-emitting diodes. Adv. Funct. Mater. 26, 4797 (2016).

9. $\mathrm{Xu}, \mathrm{B}$. et al. Bright and efficient light-emitting diodes based on MA/Cs double cation perovskite nanocrystals. J. Mater. Chem. C 5, 6123 (2017).

10. Han, T. H. et al. Interface and defect engineering for metal halide perovskite optoelectronic devices. Adv. Mater. 31, 1803515 (2019).

11. Lin, K. et al. Perovskite light-emitting diodes with external quantum efficiency exceeding 20 per cent. Nature 562, 245-248 (2018).

12. Cao, Y. et al. Perovskite light-emitting diodes based on spontaneously formed submicrometre-scale structures. Nature 562, 249-253 (2018). 
13. Zhao, B. et al. High-efficiency perovskite-polymer bulk heterostructure lightemitting diodes. Nat. Photonics 12, 783-789 (2018).

14. Zhang, L. et al. Ultra-bright and highly efficient inorganic based perovskite light-emitting diodes. Nat. Commun. 8, 1-8 (2017).

15. Okachi, T. et al. Influence of injection barrier on the determination of chargecarrier mobility in organic light-emitting diodes by impedance spectroscopy. Thin Solid Films 517, 1331-1334 (2008).

16. Wang, N. et al. Perovskite light-emitting diodes based on solution-processed self-organized multiple quantum wells. Nat. Photonics 10, 699-704 (2016).

17. Dai, X. et al. Solution-processed, high-performance light-emitting diodes based on quantum dots. Nature 515, 96-99 (2014).

18. Cho, H. et al. High-efficiency polycrystalline perovskite light-emitting diodes based on mixed cations. ACS Nano 12, 2883-2892 (2018).

19. Zou, Y. et al. Recent progress toward perovskite light-emitting diodes with enhanced spectral and operational stability. Mater. Today Nano 5, 100028 (2019).

20. Shan, Q. et al. High performance metal halide perovskite light-emitting diode: from material design to device optimization. Small 13, 1701770 (2017).

21. Gangishetty, M. K. et al. Blue perovskite LEDs: reducing architecture limitations for efficient blue perovskite light-emitting diodes. Adv. Mater. 30 , 1706226 (2018).

22. Chiba, T. et al. High-efficiency perovskite quantum-dot light-emitting devices by effective washing process and interfacial energy level alignment. ACS Appl. Mater. Interfaces 9, 18054 (2017).

23. Peng, $X$. et al. Modified conducting polymer hole injection layer for highefficiency perovskite light-emitting devices: enhanced hole injection and reduced luminescence quenching. J. Phys. Chem. Lett. 8, 4691 (2017).

24. Zeng, J. et al. Interfacial-tunneling-effect-enhanced $\mathrm{CsPbBr}_{3}$ photodetectors featuring high detectivity and stability. Adv. Funct. Mater. 29, 1904461 (2019).

25. Book, K. et al. Optical detection of charge carriers in multilayer organic lightemitting diodes: experiment and theory. J. Appl. Phys. 89, 2690-2698 (2001).

26. Thomas, J. P. et al. Defect-minimized PEDOT:PSS/planar-Si solar cell with very high efficiency. Adv. Funct. Mater. 24, 4978 (2014).

27. Sharma, B. et al. A ZnO/PEDOT:PSS based inorganic/organic hetrojunction. Solid State Commun. 149, 771-774 (2009).

28. Caruge, J. M. et al. $\mathrm{NiO}$ as an inorganic hole-transporting layer in quantumdot light-emitting devices. Nano Lett. 6, 2991 (2006).

29. Chen, $\mathrm{S}$. et al. On the degradation mechanisms of quantum-dot light-emitting diodes. Nat. Commun. 10, 765 (2019).

30. Zhang, L. et al. Capacitance-voltage characteristics of a 4,4' -bis[(N-carbazole) styryl]biphenyl based organic light-emitting diode: Implications for characteristic times and their distribution. Appl. Phys. Lett. 103, 093301.1 (2013).

31. Cai, R. et al. Perovskite light-emitting diodes based on $\mathrm{FAPb}_{1-x} \mathrm{Sn}_{x} \mathrm{Br}_{3}$ nanocrystals synthesized at room temperature. IEEE Transact. Nanotechnol. 18, 1050-1056 (2019).

\section{Acknowledgements}

This work was supported by National Key Research and Development Program (No. 2017YFE0120400), National Natural Science Foundation of China (Numbers 61875082 and 61905107), Natural Science Foundation of Guangdong (Number 2017B030306010), Key-Area Research and Development Program of Guangdong Province (Number
2019B010924001), Guangdong University Key Laboratory for Advanced Quantum Dot Displays and Lighting (Number 2017KSYS007), High Level University Fund of Guangdong Province (G02236004), Shenzhen Key Laboratory for Advanced Quantum Dot Displays and Lighting (Number ZDSYS201707281632549), and Shenzhen Innovation Project (Number JCYJ20190809152411655). W.C.H.C. acknowledges the support from the University Grant Council of the University of Hong Kong as well as the General Research Fund (Grant Numbers 17211916, 17204117, and 17200518) from Hong Kong Special Administrative Region, China.

\section{Author contributions}

X.X. and K.W. conceived the idea, designed the experiment, and analyzed the data. K.W. and W.C. supervised the work. X.X. and D.W. were responsible for sections of fielddependent hole hopping theory and simulation. T.Y. and R.C. worked on the synthesis of $\mathrm{FAPb}_{0.7} \mathrm{Sn}_{0.3} \mathrm{Br}_{3}$ perovskite nanocrystals. T.Y., Z.R., J.S., and S.D. participated in the fabrications and the characterizations measurements of PeLEDs. X.X. and X.Q. developed the low-frequency capacitance-voltage measurement for PeLEDs. X.X., K.W., and T.Y. co-wrote the manuscript. K.W., W.C., and X.S. improved the manuscript. All authors contributed to discussions and finalizing the manuscript.

\section{Competing interests}

The authors declare no competing interests.

\section{Additional information}

Supplementary information is available for this paper at https://doi.org/10.1038/s43246020-00084-0.

Correspondence and requests for materials should be addressed to K.W. or W.C.H.C.

Peer review information Primary handling editor: John Plummer.

Reprints and permission information is available at http://www.nature.com/reprints

Publisher's note Springer Nature remains neutral with regard to jurisdictional claims in published maps and institutional affiliations.

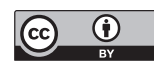

Open Access This article is licensed under a Creative Commons Attribution 4.0 International License, which permits use, sharing, adaptation, distribution and reproduction in any medium or format, as long as you give appropriate credit to the original author(s) and the source, provide a link to the Creative Commons license, and indicate if changes were made. The images or other third party material in this article are included in the article's Creative Commons license, unless indicated otherwise in a credit line to the material. If material is not included in the article's Creative Commons license and your intended use is not permitted by statutory regulation or exceeds the permitted use, you will need to obtain permission directly from the copyright holder. To view a copy of this license, visit http://creativecommons.org/ licenses/by/4.0/.

(C) The Author(s) 2020 